Classification

Physics Abstracts

$68.40-61.50 \mathrm{C}-67.80$

\title{
Observation of critical slowing down close to a roughening transition (*)
}

\author{
L. Puech, B. Hebral, D. Thoulouze and B. Castaing \\ Centre de Recherches sur les Très Basses Températures, \\ C.N.R.S., BP 166 X, 38042 Grenoble Cedex, France
}

(Reçu le 29 octobre 1982, accepté le 21 décembre 1982)

\begin{abstract}
Résumé. - Nous avons mesuré le temps de retour à une taille stable d'une facette d'un cristal de ${ }^{4} \mathrm{He}$ en lente croissance, près de la transition rugueuse de cette facette $\left(T_{\mathrm{R}} \cong 215 \mathrm{mK}\right)$. Les temps observés montrent un ralentissement critique, allant de $2 \mathrm{~min}$. loin de la transition, à $44 \mathrm{~min}$. à quelques $\mathrm{mK}$ de celle-ci.

Abstract. - We have measured the times necessary to recover the stable size of a facet of a ${ }^{4} \mathrm{He}$ crystal in a slow growing process, close to the roughening transition of the facet $\left(T_{\mathrm{R}} \cong 215 \mathrm{mK}\right)$. These times exhibit a critical slowing down from $2 \mathrm{~min}$. far from this transition to $44 \mathrm{~min}$. at a few $\mathrm{mK}$ from the transition.
\end{abstract}

1. Introduction. - Since the work of Burton, Cabrera and Frank [1], who proposed the existence of a so-called "Roughening-Transition " on the fluid-solid interface of pure compounds, studies on this subject have been essentially theoretical [2]. Its existence was first confirmid by numerical simulations [3], and then observed on real crystals [4].

The study of the interface near the roughening transition was made possible by the experiments on the interface between superfluid- and h.c.p. solid- ${ }^{4} \mathrm{He}$ [5].

We present here the first observation of critical slowing down in the vicinity of such a transition. $\mathrm{Ne}$ studied the interface between liquid and solid ${ }^{4} \mathrm{He}$ with the normal to the interface at an angle If about $70^{\circ}$ with respect to the $C$ axis of the h.c.p. structure [6] : P. E. Wolf and S. Balibar [7], tave recently observed by optical methods the appearance of a facet with the same orientation $\mathrm{t}$ around $200 \mathrm{mK}$.

2. Method of the observation. - A Kapitza resistance measurement allows us to characterize he interface, specifically the proportion of facets it contains, the facet having a smaller thermal esistance than the rough parts of the interface (which has a meniscus-like shape) [6]. The experirental cell we used is shown on figure 1, and has already been described [6]. The thermometers sed are Matsushita $68 \Omega$ Carbon resistors. In this experiment we measured their resistance ratio $?_{2} / R_{3}$ which is very sensitive to the difference $\Delta T$ in temperature between the two thermometers, ut does not depend on the mean temperature. One of them is in the solid the other is in the liquid.

*) La version française de cet article a été proposée aux Comptes Rendus de l'Académie des Sciences. 
To the mixing chamber

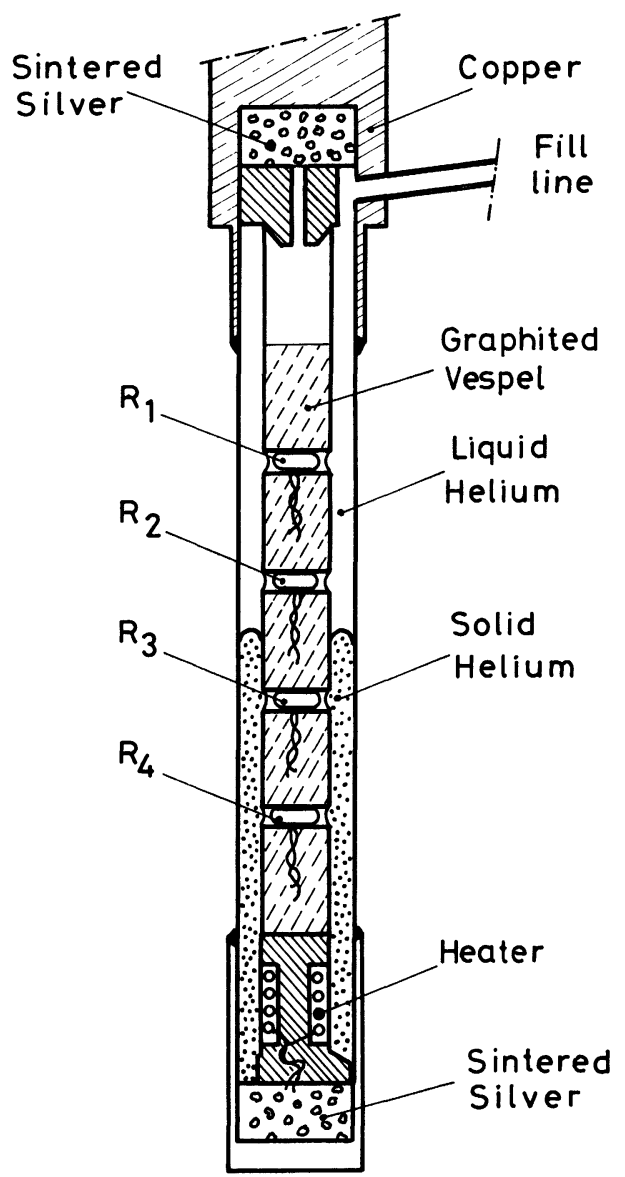

Fig. 1. - Experimental cell.

A constant heat flux of $26 \mu \mathrm{W} / \mathrm{cm}^{2}$ is maintained by an electrical heater at the bottom of the cell. The schematic diagram in figure 2 shows the principle of the measurement : having slightly distorted the interface, we observe the evolution of the ratio $R_{2} / R_{3}$.

Although the interface is always between $R_{2}$ and $R_{3}$, the evolution of the resistance ratio does not occur at constant volume of the solid. The crystal is indeed growing at a speed $V \cong 1 \mathrm{~mm} / \mathrm{h}$. In such conditions, below the roughening transition, the interface possesses a proportion of facet sufficient to reduce the Kapitza jump of an observable amount.

Superimposed on this slow growing process, we perform a perturbation of the shape of the interface by rapidly ( $\leqslant 2 \mathrm{~s}$ ) acting on the dead volume of a valve. In this way, we crystallize and then melt a small quantity of solid. The quantity of matter involved in this process corresponds to a change of $0.1 \mathrm{~mm}$ in the level of the solid. The consequence of this perturbation is to increase and suddenly decrease the size of the facet of the interface.

Thus $\Delta T$ first decreases and then increases to a value greater than the stationary value previously obtained. It then relaxes to the stationary value. The total relaxation is about $200 \mu \mathrm{K}$, which is a tenth of the total difference in temperature between the liquid and the solid. Figure 3 shows two examples of those relaxations. 


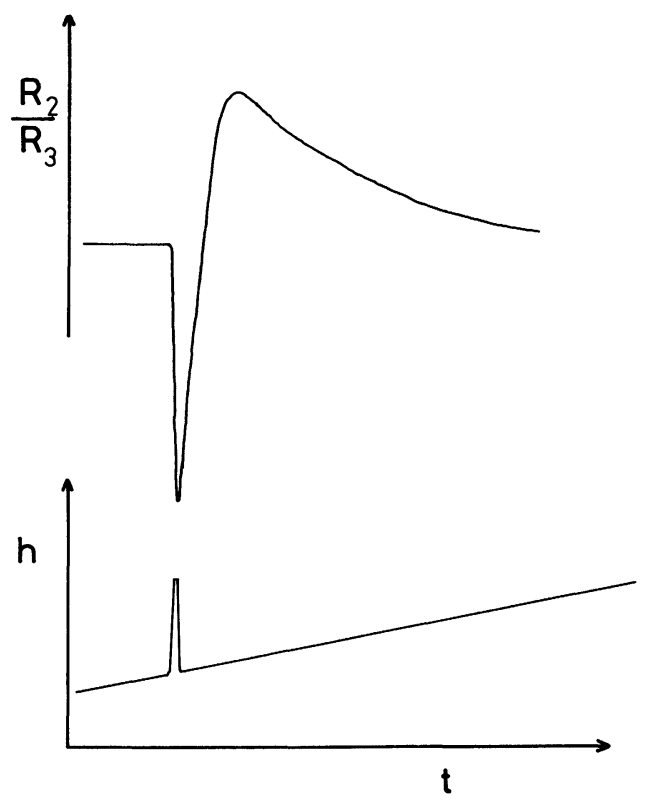

Fig. 2. - Principle of the measurement (Schematic). Time is the abscisse. The lowest trace is the height of the interface, the highest is the ratio $R_{2} / R_{3}$.

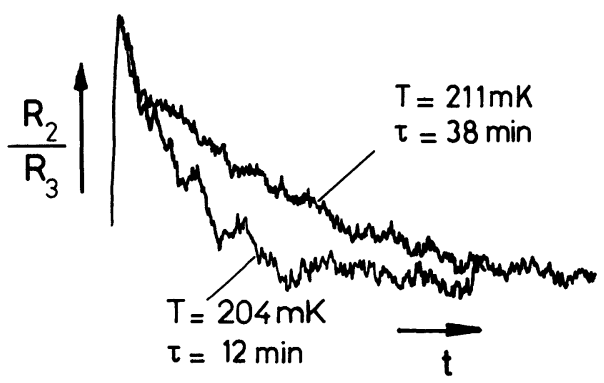

Fig. 3. - Two examples of relaxation.

3. Results and discussion. - By assimilating to an exponential function, we are able to define a time $\tau$ characteristic of this relaxation. The results are plotted in figure 4 . The base line of the relaxation is determined by the stationary size of the facet, which in turn depends strongly on the speed $V$. This speed $V$ is hard to control in practice, which gives the large scatter in the results.

Nevertheless, it is clear from figure 4 that $\tau$ diverges, when approaching a temperature that we consider as being the temperature of the roughening transition.

The shortest times presented in figure 4 are $4 \mathrm{~min}$. At lower temperature we observed times as short as $2 \mathrm{~min}$. Thus, this relaxation time varies by more than one order of magnitude from well below to near the transition. This is in agreement with the idea of a continuous transition.

Within the experimental dispersion, the results agree with a linear dependence of $1 / \tau$ versus the temperature. A «Landau-like» description of 2 nd order transitions predicts this kind of behaviour [8]. 


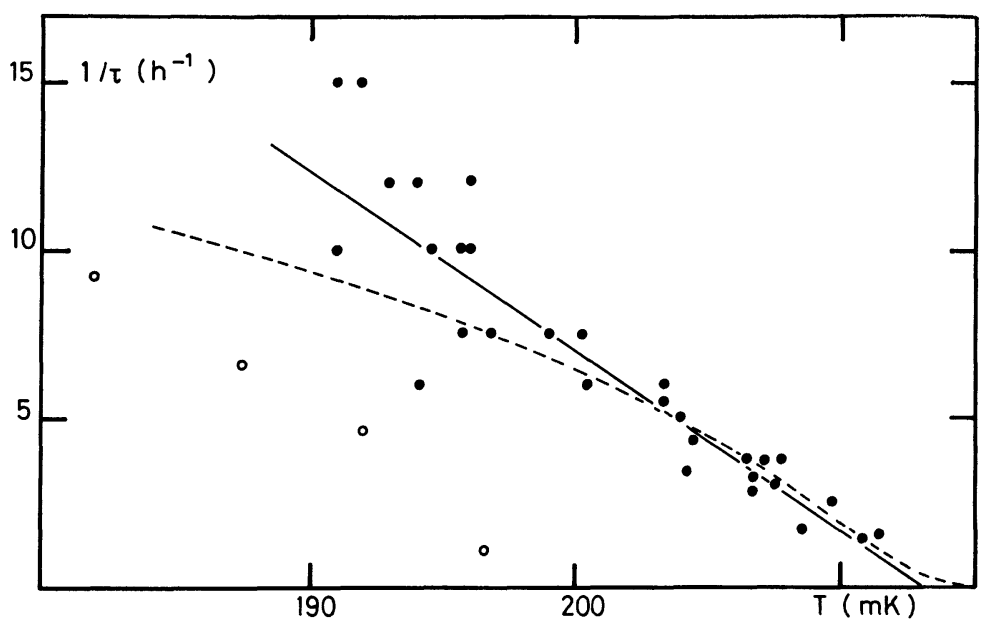

Fig. 4. $-\tau^{-1}$ as a function of temperature. $\circ$ Before annealing; $\bullet$ After annealing; $(-) \tau^{-1} \propto\left(T_{\mathrm{c}}-\right.$ $T) ;\left(--\longrightarrow \tau \propto \exp \left(\frac{b}{\left(T_{\mathrm{c}}-T\right)^{1 / 2}}\right)\right.$.

For a strictly infinite two dimensional system, such a description would certainly be wrong. However, Andreev [9] suggests that the small size of the facet limits the fluctuations, so the system could not be considered as infinite.

On the other hand, renormalization techniques, applied to the discrete Gaussian model [2] lead to a law :

$$
\tau \propto \exp \frac{b}{\left(T_{\mathrm{R}}-T\right)^{1 / 2}} .
$$

The dotted line in figure 4 corresponds to such a law. We are not able to distinguish between the theories.

Let us remark that a preliminary study gave the points (O), which lead to a quite different value of the roughening transition temperature. Between the two studies, the crystal was warmed to $0.7 \mathrm{~K}$ for $3 \mathrm{~h}$. We think that this demonstrates the sensitivity of this transition to the state of the underlying crystal [10], as pointed out by Keshishev et al. [5].

This study underlines an intriguing point : how does an annealing of the crystal influence the temperature of the transition?

A theory of the roughening transition should explain this particular feature.

4. Conclusion. - For the first time we have observed the critical slowing down close to a roughening transition, thus giving strong evidence of its continuous character. Within the experimental uncertainty, we are not able to distinguish between the mean-field theory results [9] and those obtained by renormalization techniques.

Acknowledgments. - We would like to thank M. Papoular for numerous discussions. 


\section{References}

[1] Burton, W. K., Cabrera, N. and Frank, F. C., Philos. Trans. R. Soc. 243 (1951) 299.

[2] WeEks, J. D., Ordering in Strongly Fluctuating Condensed Matter Systems, Ed. T. Riste (Plenum Press) 1980, p. 293.

[3] Leamy, H. and Gilmer, G., J. Crystal Growth 24 (1974) 499.

SWENDSEN, R. H., Phys. Rev. B 15 (1977) 5421.

[4] JACKSON, K. A. and Miller, C. E., J. Crystal Growth 40 (1977) 169.

[5] Balibar, S., Edwards, D. O. and Laroche, C., Phys. Rev. Lett. 42 (1979) 782.

Balibar, S. and Castaing, B., J. Physique Lett. 41 (1980) L-329.

Avron, J. E., Balfour, L. S., Kuper, C. G., Landau, J., Lipson, S. G. and Schulman, L. S., Phys. Rev. Lett. 45 (1980) 814.

Keshishev, K. O., Parshin, A. and Babkin, A., Sov. Phys. JeTP 53 (1981) 362.

[6] Puech, L., Hebral, B., Thoulouze, D. and Castaing, B., J. Physique-Lett. 43 (1982) L-809.

[7] Balibar, S., private communication.

[8] VAN Hove, L., Phys. Rev. 95 (1954) 1374.

See also MA, S., Modern Theory of Critical Phenomena (Benjamin) 1976, p. 442.

[9] ANDReEv, A., Sov. Phys. JETP 73 (1982) 1063.

[10] The dislocation density in our crystal was $10^{7} \mathrm{~cm}^{-2}$ in order of magnitude (see Ref. [6]). 